\title{
A crystallographic and theoretical study of an (E)-2-Hydroxyiminoethanone derivative: prediction of cyclooxygenase inhibition selectivity of stilbenoids by MM-PBSA and the role of atomic charge
}

Article

Accepted Version

Balef, S. S. H., Chippindale, A. M. and Irannejad, H. (2019) A crystallographic and theoretical study of an (E)-2Hydroxyiminoethanone derivative: prediction of cyclooxygenase inhibition selectivity of stilbenoids by MMPBSA and the role of atomic charge. Journal of Bimolecular Structure and Dynamics, 37 (6). pp. 1555-1566. ISSN 07391102 doi: https://doi.org/10.1080/07391102.2018.1462256 Available at https://centaur.reading.ac.uk/77821/

It is advisable to refer to the publisher's version if you intend to cite from the work. See Guidance on citing.

To link to this article DOI: http://dx.doi.org/10.1080/07391102.2018.1462256

Publisher: Taylor and Francis

All outputs in CentAUR are protected by Intellectual Property Rights law, including copyright law. Copyright and IPR is retained by the creators or other copyright holders. Terms and conditions for use of this material are defined in 
the End User Agreement.

www.reading.ac.uk/centaur

\section{CentAUR}

Central Archive at the University of Reading

Reading's research outputs online 


\section{A crystallographic and theoretical study of an (E)-2-Hydroxyiminoethanone derivative: prediction of cyclooxygenase inhibition selectivity of stilbenoids by MM-PBSA and the role of atomic charge}

Seyed Sajad Hosseini Balef ${ }^{1}$, Ann M. Chippindale ${ }^{2}$, Hamid Irannejad*1

1 Department of Medicinal Chemistry, Faculty of Pharmacy, Mazandaran University of Medical Sciences, Sari, Iran

2 Department of Chemistry, University of Reading, Whiteknights, Reading, Berks RG6 6AD, UK

We recently reported that the hydroxyiminoethanone derivative, $(E)-\mathbf{O X M}$, behaves as a highly selective COX-1 inhibitor (COX-1 SI= 833), and also an interesting scaffold with unique characteristics. In the current study, a comprehensive crystallographic and computational study was performed to elucidate its conformational stability and pharmacological activity. Its conformational energy was studied at the B3LYP/6-311G** level of theory and compared to the single-crystal X-ray diffraction data. In addition, computational studies of three structurally different stilbenoid derivatives used as selective COX-1 or COX-2 inhibitors were undertaken to predict their COX selectivity potentials. Flexible docking was performed for all compounds at the active site of both COX-1 and COX-2 enzymes by considering some of the key residues as flexible during the docking operation. In the next step, molecular dynamic simulation and binding free energy calculations were performed by MM-PBSA. Final results were found to be highly dependent on the atomic charges of the inhibitors and the choice of force field used to calculate the atomic charges. The binding conformation of the hydroxyiminoethanone derivative is highly correlated with the type of COX isoform inhibited. Our predictive approach can truly predict the cyclooxygenase inhibition selectivity of stilbenoid inhibitors.

Keywords: X-ray crystallography, MM-PBSA, Atomic charge, Cyclooxygenase inhibition selectivity, Hydroxyiminoethanone, Stilbenoid

*Correspondence: Dr. Hamid Irannejad, Department of Medicinal Chemistry, Faculty of Pharmacy, Mazandaran University of Medical Sciences, Sari, Iran. E-mail: irannejadhamid@ gmail.com, Phone: 0098-11-33543082, Fax: 0098-11-33543084 


\section{Introduction}

Cyclooxygenase (COX) is a key enzyme associated with inflammation and cell injury, which are processes in which many inflammatory mediators are responsible for cell degeneration. Prostaglandins are important inflammatory mediators produced by cyclooxygenase and have various functions in different tissues, for example, suppression of fever in the central nervous system, platelet aggregation and so on. Neuroinflammation is responsible for neurodegerative diseases, such as Alzheimer's and Parkinson's, in which COX isoforms (COX-1 and COX-2) play a vital role in the initiation and progression of the diseases (Kaur, Gill, Bansal, \& Deshmukh, 2017).

Cyclooxygenase has two isoforms, namely COX-1 and COX-2, which are active in normal and pathologic conditions, respectively, in specific tissues. Many studies have shown that COX-1 and COX-2 play major roles in the progression of neurological situations initiated by neuroinflammation. Selective inhibition of COX-1 has been shown to slow down in the first stages of progressive Alzheimer's disease (Barbalho, Carvalho, Lopes-Cendes, \& MaurerMorelli, 2016; Calvello et al., 2017; Choi et al., 2013). Moreover, selective COX-2 inhibitors have been administered to reduce brain cytokine levels following seizures (Temp et al., 2017). Therefore, selectivity in the inhibition of COX isoforms is very important. One of the main factors influencing COX inhibition selectivity is the conformation of the inhibitor and its conformational flexibility (Irannejad et al., 2015). Only a few studies have been reported in the literature which consider the conformation of the inhibitor and its selectivity towards COX inhibition. This structure-property relationship has been explored significantly in stilbenoid COX inhibitors, in which two aryl rings are attached through an olefinic bond, or any linkage which has some degree of rigidity, and favor a preferred stable conformation (Kakuta et al., 2008; Uddin, Rao, Rahim, McDonald, \& Knaus, 2004; Zheng et al., 2007). Therefore, there is a substantial need to perform a comprehensive and extensive study of the relationship between the binding conformation and COX inhibition selectivity of stilbene-like structures.

Recently, we unexpectedly discovered that a 2-hydroxyiminoethanone derivative, $(E)$-OXM, behaves as a highly selective COX-1 inhibitor (IC50 COX-1=0.12 $\mu \mathrm{M}$, IC50 COX-2 >100 $\mu \mathrm{M}$, SI >833). This molecule also contains a sulfonylmethyl group which is a COX-2 selective pharmacophoric element (Figure 1) (Irannejad, et al., 2015). In the same report, a preliminary 
computational study was performed to compare two states of the molecule, either when rigid or flexible, for docking. The results showed that for selective COX-1 inhibition, the molecule has to adopt both cisoid and transoid conformations, but for selective COX-2 inhibition only the cisoid conformation of the molecule is necessary for binding to the active site of COX-2. Since many aspects of COX selective inhibition remain unknown and also several research articles have reported similar results with stilbenoid structures containing amide, sulfonamide and olefinic units (Figure 2) (Kakuta, et al., 2008; Uddin, et al., 2004; Zheng, et al., 2007), we decided to do a comprehensive in-silico study to establish the relationship between conformation and COX selectivity.

In this extended study, we studied computationally a 2-hydroxyiminoethanone, (E)-OXM, together with three different stilbenoids, AMID, SULF and HEXL, previously reported in the literature as $\mathrm{COX}$ inhibitors (Figure 2). The 2-hydroxyiminoethanone molecule was studied structurally by X-ray crystallography to determine its most stable conformation in the solid state and then it was optimized at the level of B3LYP/6-311G**. In the next stage, the compounds AMID, SULF and HEXL (Figure 2) were flexibly docked into the active sites of COX-1/2, while key residues were considered flexible in the enzyme pocket. The best binding conformation of each compound was then extracted and input in to molecular dynamic simulation for $500 \mathrm{ps}$. The free energy of binding for each compound was then calculated and compared for both isoforms utilizing the MM-PBSA method.

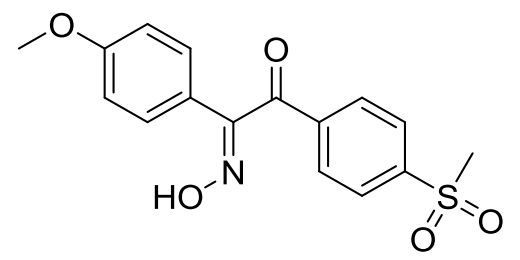

Figure 1. Compound (E)-OXM which was recently reported as a highly selective COX-1 inhibitor (IC50 COX-1=0.12 $\mu \mathrm{M}$, IC50 COX-2 >100 $\mu \mathrm{M}$, SI >833). 


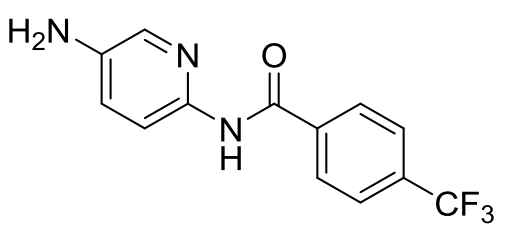

(a)

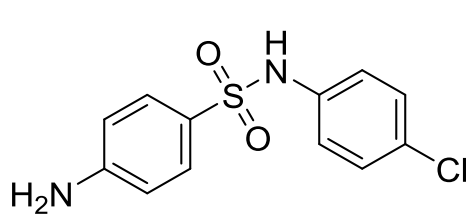

(b)

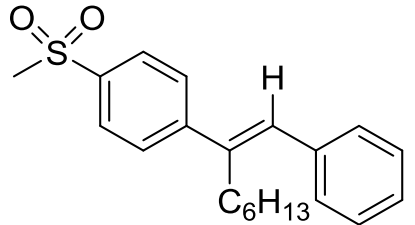

(c)

Figure 2. Stilbenoid compounds a) AMID (COX-1 selective, IC50 COX-1=0.8 $\mu$ M, IC50 COX-2 >100 $\mu \mathrm{M})$, b) SULF (COX-1 selective, IC50 COX-1= $3.2 \mu \mathrm{M}$, IC50 COX-2 >100 $\mu \mathrm{M})$, c) HEXL (COX-2 selective, IC50 COX-1 >100 $\mu \mathrm{M}$, IC50 COX-2 = $0.77 \mu \mathrm{M})$.

\section{Methods}

\section{Conformational analysis}

A conformational search was performed by Gaussian 03 with PM3 force field for the two dihedral angles, O9-C8-C10-N11 and H-O12-N11-C10 which were scanned in 40 steps of 10 degrees and 20 steps of 20 degrees, respectively. The best conformer with the lowest energy was extracted and used for geometry optimization by DFT calculations at the level of B3LYP/6$311 \mathrm{G}^{* *}$ in both vacuum and water.

\section{Flexible docking}

PDB structure of COX-1/2 isoforms were retrieved from www.rcsb.org with 1Q4G and 3NT1 codes and used in the whole procedure. Docking was performed by AutoDock 4 and residues Arg120, Tyr385 and Ser530 in COX-1 and His90, Arg120, Arg513 and Ser530 in Cox-2 were selected to be flexible during the docking simulation (Chaudhary and Aparoy, 2017). The other parameters used were the following: Grid center in COX-1: $X=24.917, Y=34.602, Z=201.23$ and in COX-2: $\mathrm{X}=-43.58, \mathrm{Y}=-52.702, \mathrm{Z}=-19.287$, Grid spacing: 0.375 , number of points: $60 \mathrm{x}$ 60 x 60, number of evaluations: 2500000, population size: 150, GA run: 20. The best docking pose with the lowest free energy of binding was extracted and chosen for dynamic simulation and free energy calculation (Dadashpour et al., 2015). 2D images of ligand-receptor interactions were prepared by Discovery Studio Visualizer 4.5.

\section{Molecular dynamic simulation \& Binding free energy calculation}

GROMACS 5.0 was used for the dynamic simulation of compounds in the COX isoforms for $500 \mathrm{ps}$ in explicit water and the gromos96 43A1 force field was used for topology generation. 
The topology of the ligands was generated by the PRODRG online server and the atomic charges were modified using AM1-BCC, calculated by antechamber program of AmberTools 12, or PM3 and Mulliken atomic partial charges, calculated by Gaussian 09 (Frisch et al., 2009). The topology and coordinate files for the protein were generated using the pdb2gmx program of the GROMACS package taking parameters from the gromos96 43A1 force field. The coordinate and topology files of the protein and the ligands were then merged to obtain the final starting structure and topology file for each complex.

The complex was centered in a dodecahedral periodic box and solvated by the addition of TIP3P water molecules. The total charge of the system was then neutralized by addition of sodium and chloride ions as required. Sequentially, energy minimization was performed by using the steepest descent algorithm. The system was then gradually heated to $300 \mathrm{~K}$ and was equilibrated for 200 ps using the NVT (constant volume and temperature) ensemble with position restraints applied to the heavy atoms followed by 200 ps equilibration in the NPT (constant pressure and temperature) ensemble at $1 \mathrm{~atm}$. Both temperature and pressure were regulated using the Berendsen algorithm. Finally, the full system was subjected to 500 ps MD simulation with a 2 fs time step. The temperature and pressure were maintained at $300 \mathrm{~K}$ and $1 \mathrm{~atm}$, respectively, using the v-rescale temperature and the Parrinello-Rahman pressure coupling method. The short-range non-bonded interactions were computed for the atom pairs within the cut-off of $1 \mathrm{~nm}$, while the long-range electrostatic interactions were calculated using the Particle-Mesh-Ewald summation method with

fourth-order cubic interpolation and 1.2 $\AA$ grid spacing. All bonds were constrained using the parallel LINCS method.

Finally, the MM-PBSA method for calculating free energies of binding was carried out using the g-mmpbsa script introduced by Rashmi Kumari (Kumari, Kumar, Open Source Drug Discovery, \& Lynn, 2014). The g-mmpbsa tool is an open-source tool written in the $\mathrm{C}$ programming language and does not depend on any external software. This tool contains all the required subroutines from the GROMACS and the APBS packages to calculate the enthalpic components of the MM-PBSA interaction (Singh, Awasthi, Pandey, \& Dwivedi, 2017).

\section{X-ray structure determination details}

Compound (E)-OXM was prepared according to our previous published article (Irannejad, et al., 2015). Single crystals of this compound were grown by slow evaporation of the ethanol solvent. 
A crystal of (E)-OXM was mounted under Paratone-N oil and flash cooled to $150 \mathrm{~K}$ in a stream of nitrogen in an Oxford Cryostream cooler. Single-crystal X-ray intensity data (Table 1) were collected using a Rigaku Gemini S Ultra diffractometer (Mo K $\alpha$ radiation $(\lambda=0.71073 \AA$ )). The data were reduced within the CrysAlisPro software (Rigaku, Xcalibur/SuperNova CCD System, $\&$ formerly Oxford Diffraction, 2012). The structure was solved in the triclinic space group, $P-1$, using the program Superflip (Palatinus and Chapuis, 2007) and all nonhydrogen atoms located. Least-squares refinements on $F$ were carried out using the CRYSTALS suite of programs (Betteridge, Carruthers, Cooper, Prout, \& Watkin, 2003). The nonhydrogen atoms were refined anisotropically. Hydrogen atoms could all be located in difference Fourier maps. The hydrogen atom in the $\mathrm{O}(12)-\mathrm{H}(1)$ hydroxide group was refined isotropically. The remaining hydrogens were placed geometrically with a C-H distance of $0.95 \AA$ and a Uiso of 1.2 times the value of Ueq of the parent $\mathrm{C}$ atom and their positions refined with riding constraints. There is one molecule of (E)-OXM in the asymmetric unit, giving rise to two molecules in the unit cell. Data have been deposited at the Cambridge Crystallographic Data Centre, deposit code: CCDC 1566249.

\section{Results \& discussion}

The ORTEP structure and crystallographic data of compound (E)-OXM are presented in Figure 3 and Table 1 , respectively, and clearly indicate the $E$-isomeric form of the imine bond in this molecule.

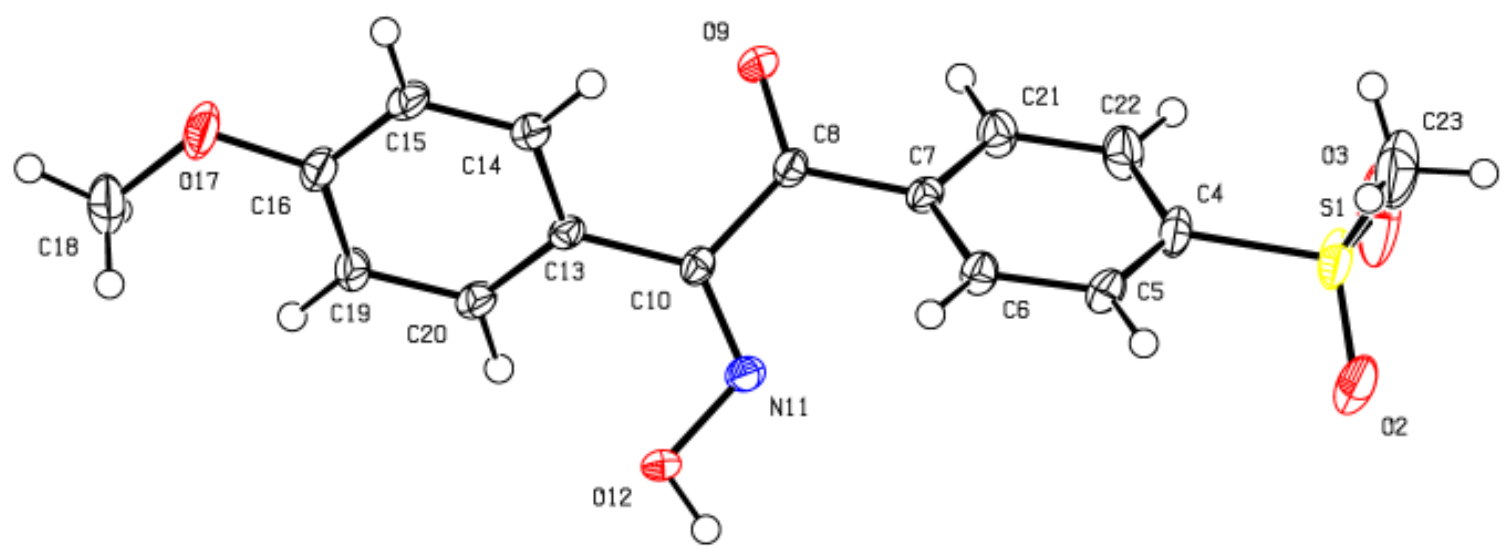

Figure 3. ORTEP structure of compound (E)-OXM (thermal ellipsoids shown at 50\% probability); Asymmetric unit with labelling scheme. 
Table 1. Crystallographic and structure refinement data of compound (E)-OXM at $150 \mathrm{~K}$.

\begin{tabular}{ll}
\hline Formula & $\mathrm{C}_{16} \mathrm{H}_{15} \mathrm{~N} \mathrm{O}_{5} \mathrm{~S}$ \\
$M_{\mathrm{r}}$ & 333.365 \\
Crystal System & Triclinic \\
Space group & $P-1$ \\
$a / \AA$ & $6.4993(4)$ \\
$b / \AA$ & $7.2813(7)$ \\
$c / \AA$ & $16.7732(12)$ \\
$\alpha /{ }^{\circ}$ & $98.093(7)$ \\
$\beta /{ }^{\circ}$ & $95.852(5)$ \\
$\gamma /{ }^{\circ}$ & $90.002(6)$ \\
Volume / $\AA^{3}$ & $781.68(11)$ \\
$\mathrm{D}_{\text {calc }} / \mathrm{g} \mathrm{cm}^{3}$ & 1.416 \\
$\mathrm{Crystal} \mathrm{Habit}$ & yellow plate \\
No. Reflections $(I \leq 3 \sigma(I))$, no. & 2651,212 \\
parameters & \\
R factors: R, Rw $(\%)$ & $5.02,5.79$ \\
\hline
\end{tabular}

The bond lengths and angles in the molecule are as expected for the particular organic groups and are given in Tables S2 and S3 in the supplementary material. The torsion angle, C(13)$\mathrm{C}(10)-\mathrm{C}(8)-\mathrm{C}(7)$, is $172.8(2)^{\circ}$. The angle between the mean planes of the two benzene rings is $\sim 87^{\circ}$. Molecules stack on top of each other along the $a$ axis in the packed structure. Hydrogen bonding interactions between adjacent molecules $\mathrm{O}(12)-\mathrm{H}(1)$...O(9), 2.710(3) $\AA$, are clearly seen when the packed structure is viewed along the $c$ axis (Figure 4). Similar values for bond lengths and dihedral angles have been reported for symmetrical benzyl monoximes (Klein, Fischer, Seichter, Schwarzer, \& Weber, 2011). 

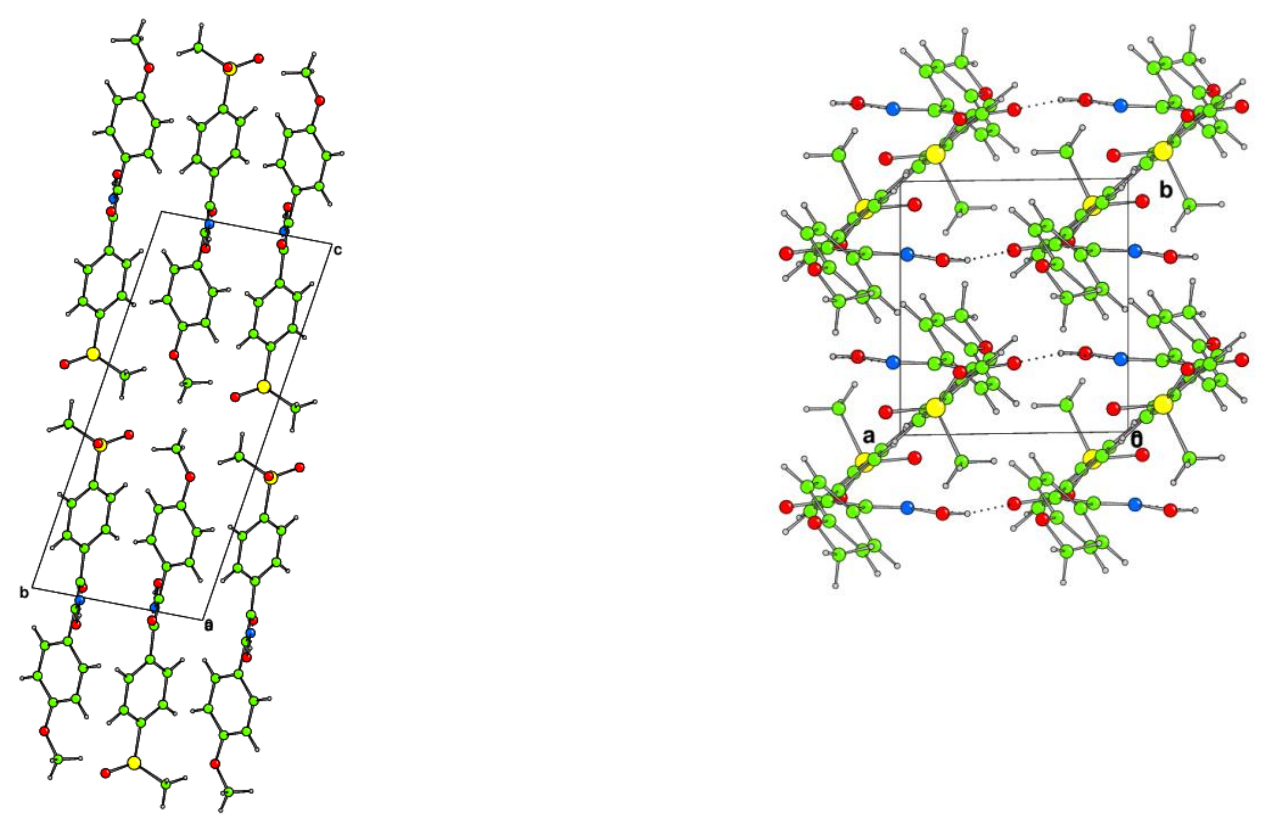

Figure 4. a) View of unit cell of (E)-OXM along the $a$ axis. b) View of unit cell along $c$ axis. Hydrogen bonding interactions are shown as dotted lines.

In order to better understand the conformational stability of $(E)$-OXM in various situations, conformational analysis was performed for the two important dihedral angles, O9-C8-C10-N11 and H-O12-N11-C10. For this purpose, O9-C8-C10-N11 was scanned every 10 degrees for 40 steps and H-O12-N11-C10 was scanned for 20 steps of each 20 degrees. In total, 861 conformations $(41 \times 21)$ were generated and scanned for their energy values and their $3 \mathrm{D}$ graphical representation is illustrated in Figure 5, as a potential energy surface diagram. The best conformer with the lowest energy as a global minimum ( $\mathrm{E}=-0.12049$ a.u.) with dihedral angles of $\mathrm{O} 9-\mathrm{C} 8-\mathrm{C} 10-\mathrm{N} 11=111^{\circ}$ and $\mathrm{H}-\mathrm{O} 12-\mathrm{N} 11-\mathrm{C} 10=160^{\circ}$ was selected for the next step, i.e. Geometry optimization by DFT calculations at the level of B3LYP/6-311G** in water and vacuum. 


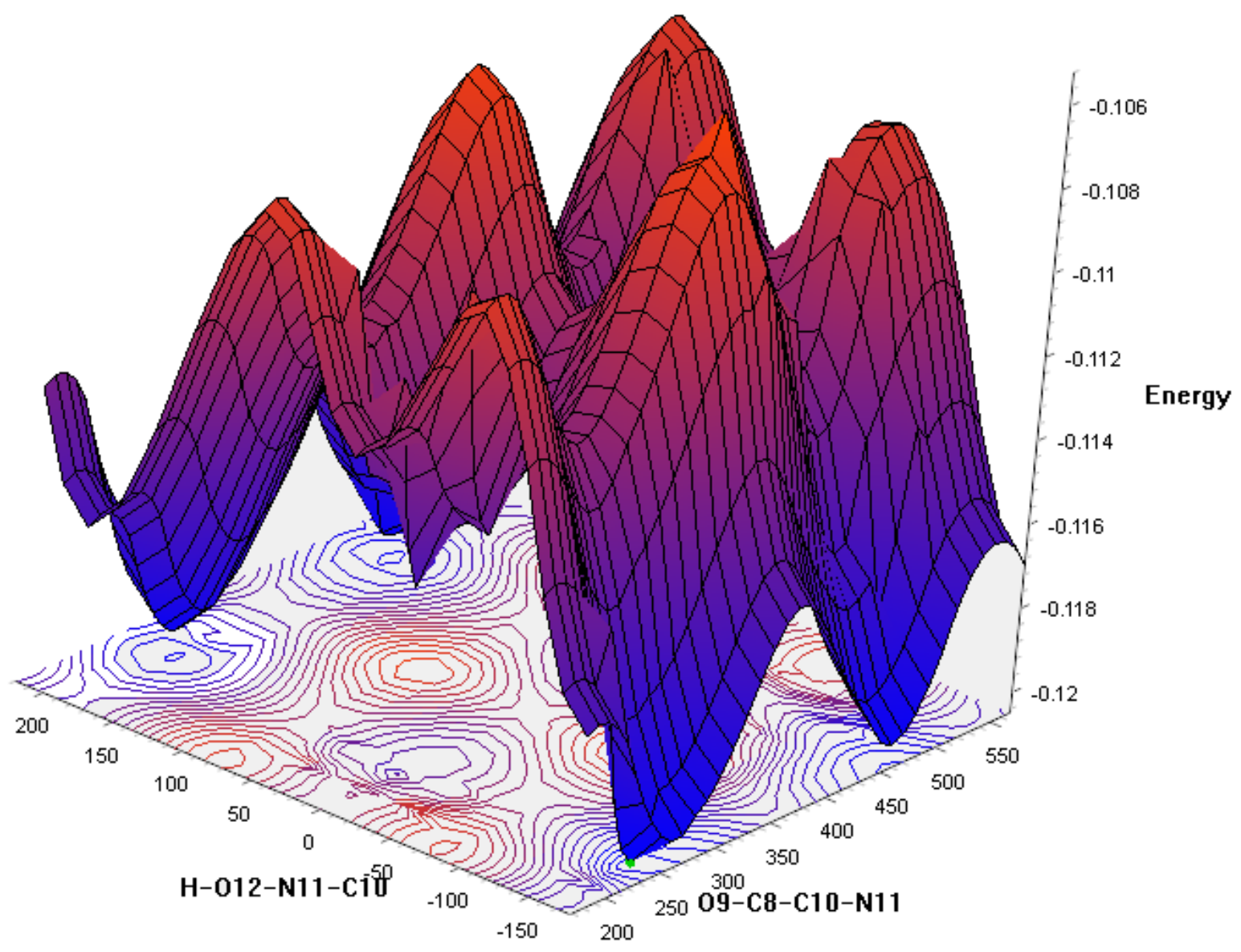

Figure 5. Potential energy surface scan of (E)-OXM for the two dihedral angles, O9-C8-C10N11 and H-O12-N11-C10.

The results of geometry optimization, i.e. output conformations, dihedral angles and their energies are summarized in Table 2. As seen in the table, the resulting optimized conformation of $(E)-\mathbf{O X M}$ in vacuum and in aqueous solution are transoid and the O9-C8-C10-N11 dihedral angle $\left(146^{\circ}\right.$ and $151^{\circ}$ in vacuum and water) has become much larger compared to the corresponding angle in the input structure $\left(111^{\circ}\right)$. The torsion angle, $\mathrm{C}(13)-\mathrm{C}(10)-\mathrm{C}(8)-\mathrm{C}(7)$, is $156^{\circ}$ in the optimized structure in water and $150^{\circ}$ in the optimized structure in vacuum. These data show that the computationally optimized $(E)$-OXM structure in water is very similar to its crystallized structure. To resolve this similarity in conformation, the two structures were aligned and their RMSD was calculated to be 0.174 , which confirms the close similarity between the two structures (Figure 6). 
Table 2. Geometry optimization results for $(E)$-OXM at the level of B3LYP/6-311G** in water and vacuum. Input structure for optimization was based on the results of a potential energy surface scan of the two dihedral angles.

\begin{tabular}{|c|c|c|}
\hline \multirow{2}{*}{$\begin{array}{l}\text { Input conformation } \\
\text { (O9-C8-C10-N11 } \\
\text { dihedral angle) }\end{array}$} & \multicolumn{2}{|c|}{$\begin{array}{c}\text { Output conformation (O9-C8-C10-N11 dihedral angle) } \\
\text { energy (au) }\end{array}$} \\
\hline & in water & in vacuum \\
\hline (E)-OXM & Transoid $\left(151^{\circ}\right)$ & Transoid $\left(146^{\circ}\right)$ \\
\hline$\left(111^{\circ}\right)$ & -1447.9789865 & -1447.9550161 \\
\hline
\end{tabular}

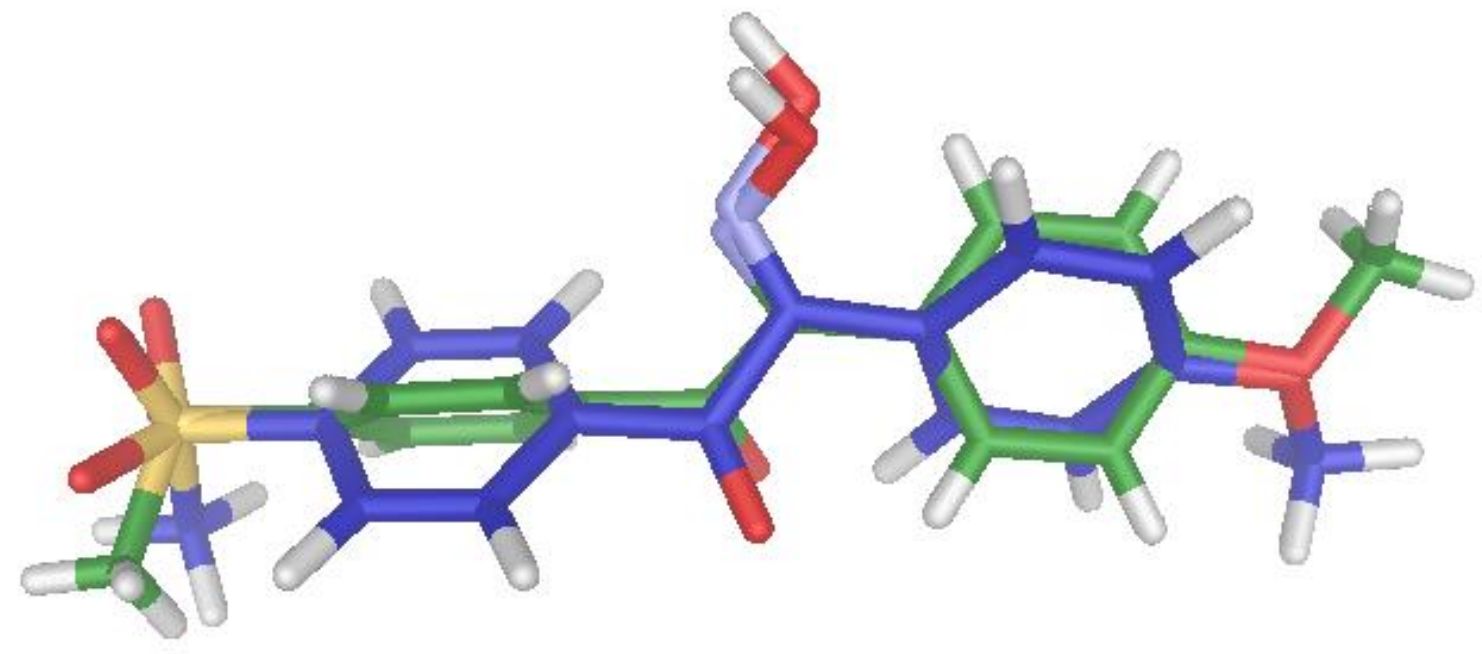

Figure 6. Alignment of the two molecules for RMSD calculation (RMSD $=0.174)$. Blue: computationally optimized $(E)$-OXM structure, Green: crystallized $(E)$-OXM structure.

Flexible docking was performed for $(E)$-OXM and also for the compounds SULF, AMID and HEXL in the active sites of both COX-1 and COX-2 enzymes. Residues Arg120, Tyr385 and Ser530 in COX-1 and His90, Arg120, Arg513 and Ser530 in COX-2 were selected to be flexible during docking simulation and the results are summarized in Table 3. It is shown, that of the compounds docked into the active sites of both isoenzymes, $(E)-\mathbf{O X M}$ is the most selective COX-1 inhibitor $\left(\Delta \mathrm{G}_{\mathrm{COX}-1}=-9.85, \Delta \mathrm{G}_{\mathrm{COX}-2}=-8.43 \mathrm{kcal} / \mathrm{mol}\right)$. These results confirm our experimentally determined $\mathrm{IC}_{50}$ and selectivity index reported previously for this compound 
(Irannejad, et al., 2015). Data obtained for the other compounds, SULF, HEXL and AMID, are also compatible with the experimentally determined COX selectivity values reported in the literature. Compounds SULF and AMID have been reported to be selective COX-1 inhibitors and HEXL was shown to be a selective COX-2 inhibitor (Kakuta, et al., 2008; Uddin, et al., 2004; Zheng, et al., 2007). As seen in Table 3, the calculated binding energy values for SULF and AMID support their COX-1 selectivity and those for compound HEXL demonstrate its COX-2 selective inhibition.

Table 3. Binding energy data $(\mathrm{kcal} / \mathrm{mol})$ of flexible docking of compounds into the active site of COX-1 and COX-2.

\begin{tabular}{ccccc}
\hline Enzyme & $(E)-$ OXM & SULF & AMID & HEXL \\
\hline COX-1 & -9.85 & -8.92 & -6.94 & -8.07 \\
COX-2 & -8.43 & -7.98 & -6.70 & -9.84 \\
\hline
\end{tabular}

The interacting amino acids with (E)-OXM, SULF, AMID and HEXL in the active sites of COX-1 or COX-2 are illustrated in Figure 7. As shown in this figure, Tyr385 and Ser530 in the active site of COX-1 are involved in hydrogen bonding to the oxygen atom of sulfonyl group in (E)-OXM. There are also several pi-alkyl or pi-pi interactions between the hydrophobic amino acids (Val349, Val116, Ala527, Leu352, Ile523, Phe531 and Tyr355) and (E)-OXM. Interestingly, Arg120 does not have any interactions with $(\boldsymbol{E})-\mathbf{O X M}$ and the hydroxyimino and carbonyl groups of $(\boldsymbol{E})-\mathbf{O X M}$ are not bonded to any atoms of the amino acids. Notably, the binding conformation of this molecule is transoid and the torsion angle, O9-C8-C10-N11 is $139^{\circ}$. Interacting amino acids of COX-1 active site with AMID and SULF are illustrated in Figures 7B and 7C. Most of the bonds formed between COX-1 and AMID and SULF are naturally hydrophobic originating from non-polar amino acids. Typically, pi-pi or pi-alkyl interactions are formed by Leu531, Val116, Val349, Tyr355, Leu384, Phe381 and Ile523 and the two inhibitors are in transoid binding conformations.

The binding conformation of HEXL as a COX-2 selective inhibitor is illustrated in Figure 7D. As shown and expected, the sulfonyl group of this molecule is inserted in the allosteric pocket 
and is hydrogen bonded to His90, Phe518 and Ala516. The other interactions are mostly hydrophobic by non-polar or aromatic amino acids.
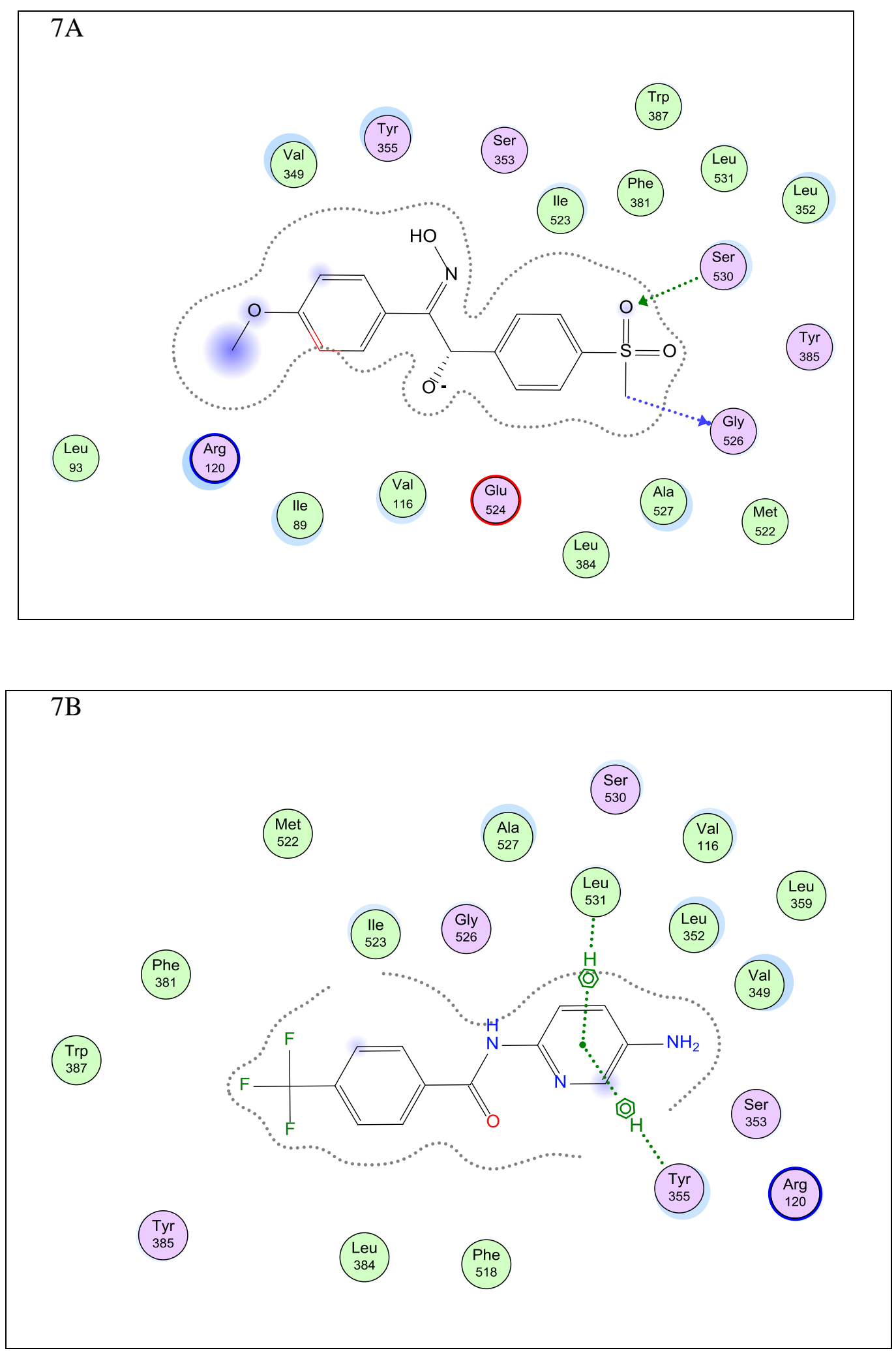

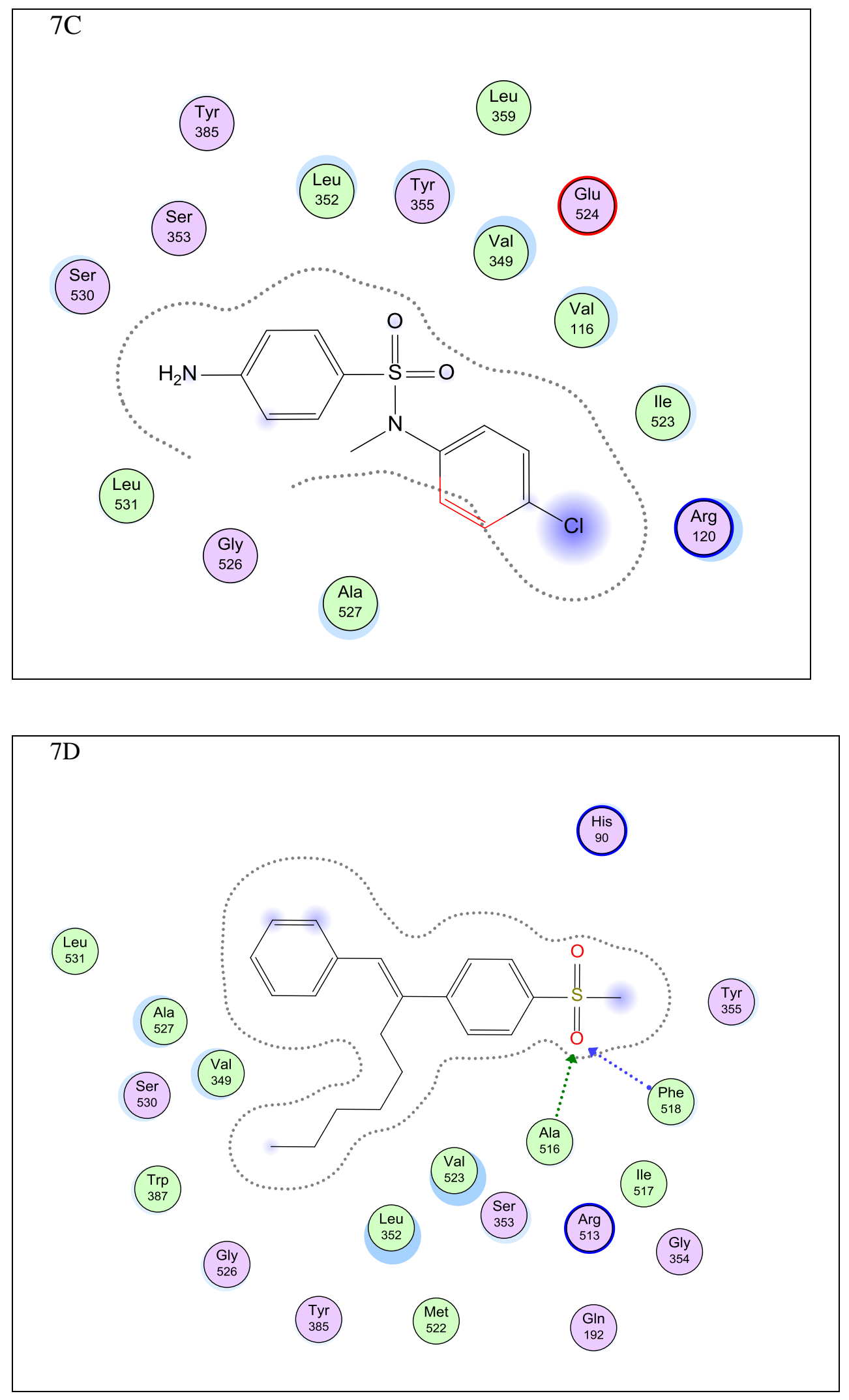
Figure 7. 2D image of interacting amino acids with (E)-OXM, SULF, AMID and HEXL with COX1/2. A) (E)-OXM in the active site of COX-1. B) SULF in the active site of COX-1. C) AMID in the active site of COX-1. D) HEXL in the active site of COX-2.

Table 4. Binding energy data of compound $(E)-\mathbf{O X M}$ in $\mathrm{kJ} / \mathrm{mol}$ calculated by MM-PBSA in the active site of COX-1 and COX-2. Atomic charges calculated by B3LYP/6-311G**.

\begin{tabular}{cccccc}
\hline Enzyme & vdw & Electrostatic & polar solvation & SASA & $\begin{array}{c}\text { Mean Binding } \\
\text { energy }\end{array}$ \\
\hline COX-1 & -183.26 & -91.60 & 266.527 & -18.39 & -26.72 \\
COX-2 & -180.26 & -204.35 & 372.23 & -17.86 & -30.27 \\
\hline
\end{tabular}

Table 5. Binding energy data of compounds in $\mathrm{kJ} / \mathrm{mol}$ calculated by MM-PBSA in the active site of COX-1. Atomic charges calculated by AM1-BCC.

\begin{tabular}{cccccc}
\hline Compound & vdw & Electrostatic & polar solvation & SASA & $\begin{array}{c}\text { Mean Binding } \\
\text { energy }\end{array}$ \\
\hline$(E)$-OXM & -234.12 & -51.15 & 192.35 & -19.31 & -112.2 \\
AMID & -171.35 & -16.39 & 93.72 & -15.94 & -109.98 \\
SULF & -164.54 & -67.15 & 126.47 & -17.57 & -122.79 \\
\hline
\end{tabular}

Table 6. Binding energy data of compounds in $\mathrm{kJ} / \mathrm{mol}$ calculated by MM-PBSA in the active site of COX-2. Atomic charges calculated by AM1-BCC.

\begin{tabular}{cccccc}
\hline Compound & $\mathrm{vdw}$ & Electrostatic & polar solvation & SASA & $\begin{array}{c}\text { Mean Binding } \\
\text { energy }\end{array}$ \\
\hline$(E)$-OXM & -204.68 & -0.597 & 140.319 & -19.031 & -83.9 \\
AMID & -173.20 & -23.76 & 91.26 & -17.31 & -123.02 \\
SULF & -178.95 & -79.31 & 131.72 & -19.51 & -146.05 \\
\hline
\end{tabular}


Table 7. Binding energy data of compounds in $\mathrm{kJ} / \mathrm{mol}$ calculated by MM-PBSA in the active site of COX-2. Atomic charges calculated by PM3.

\begin{tabular}{cccccc}
\hline Compound & vdw & Electrostatic & polar solvation & SASA & $\begin{array}{c}\text { Mean Binding } \\
\text { energy }\end{array}$ \\
\hline SULF & -157.97 & -17.66 & 93.78 & -16.115 & -97.97 \\
AMID & -187.60 & 0.67 & 64.41 & -16.67 & -139.19 \\
HEXL & -153.12 & -152.63 & 117.85 & -15.88 & -203.79 \\
(E)-OXM & -177.31 & -58.10 & 109.87 & -17.18 & -142.04 \\
\hline
\end{tabular}

Table 8 . Binding energy data of compounds in $\mathrm{kJ} / \mathrm{mol}$ calculated by MM-PBSA in the active site of COX-1. Atomic charges calculated by PM3.

\begin{tabular}{cccccc}
\hline Compound & vdw & Electrostatic & polar solvation & SASA & $\begin{array}{c}\text { Mean Binding } \\
\text { energy }\end{array}$ \\
\hline SULF & -160.08 & -42.00 & 95.42 & -17.40 & -124.06 \\
AMID & -191.23 & -11.02 & 55.22 & -16.12 & -163.15 \\
HEXL & -227.55 & -106.13 & 154.47 & -20.62 & -199.84 \\
$(E)$-OXM & -232.28 & -37.45 & 106.98 & -18.95 & -181.70 \\
\hline
\end{tabular}

In the next step, the best docked conformation of each molecule with lowest energy was extracted from the flexible docking procedure into the active sites of the COX-1 and COX-2 enzymes. The top ranked conformation was inputted into a molecular dynamic simulation as an enzyme-inhibitor complex for 500 ps. Atomic charges were calculated by AM1-BCC, PM3 and B3LYP/6-311G** and all steps of dynamic simulation and binding free energy calculations were performed by mm-pbsa method introduced by Rashmi Kumari (Kumari, et al., 2014). A brief description of this method is helpful to understand the relationships between the physicochemical parameters used in the method. Essentially, the binding free energy of a complex is calculated from the following equation: 
$\Delta \mathrm{G}_{\text {binding }}=\mathrm{G}_{\text {complex }}-\left(\mathrm{G}_{\text {protein }}+\mathrm{G}_{\text {ligand }}\right)$

Where, $\mathrm{G}_{\text {complex }}$ is the total free energy of the protein-ligand complex and $\mathrm{G}_{\text {protein }}$ and $\mathrm{G}_{\text {ligand }}$ are the total free energies of the isolated entities.

For each individual entity $(\mathrm{X})$, free energy can be calculated by:

$\mathrm{G}_{\mathrm{x}}=\left(\mathrm{E}_{\mathrm{MM}}\right)-\mathrm{TS}+\left(\mathrm{G}_{\text {solvation }}\right)$

Where, $\mathrm{E}_{\mathrm{MM}}$ is the average molecular mechanics potential energy in vacuum. TS denotes the product of the temperature and the entropic contribution and $\mathrm{G}_{\text {solvation }}$ is the free energy of solvation. In addition,

$\mathrm{E}_{\mathrm{MM}}=\mathrm{E}_{\text {bonded }}+\mathrm{E}_{\text {non-bonded }}=\mathrm{E}_{\text {bonded }}+\left(\mathrm{E}_{\mathrm{vdw}}+\mathrm{E}_{\text {elec }}\right)$

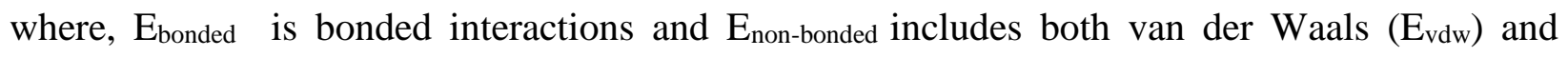
electrostatic (E Eece)interactions and are modeled using a Lennard-Jones (LJ) and Coulomb potential function, respectively.

$\mathrm{G}_{\text {solvation }}$ (solvation free energy) is expressed in the following equation:

$\mathrm{G}_{\text {solvation }}=\mathrm{G}_{\text {polar }}+\mathrm{G}_{\text {non-polar }}$

In this equation, $G_{\text {polar }}$ and $G_{\text {non-polar }}$ are the electrostatic and non-electrostatic contributions to the solvation free energy, respectively. $\mathrm{G}_{\text {non-polar }}$ includes attractive and repulsive forces between solvent and solute that are generated by van der Waals' interactions and cavity formation, respectively.

$\mathrm{G}_{\text {non-polar }}=\mathrm{G}_{\text {cavity }}+\mathrm{G}_{\mathrm{vdw}}$

One of the most widely used non-polar models to estimate the free energy of solvation is the solvent accessible surface area (SASA).

Finally, mean binding energy, average van der Waals' and electrostatic energies as well as polar solvation and SASA non-polar energies were calculated for each compound in both COX isoforms.

Firstly, Mulliken and partial atomic charges calculated at the B3LYP/6-311G** level were used for $(E)$-OXM in a dynamic simulation and binding-energy calculation. The obtained results were not favorable and satisfactory since lower $\Delta \mathrm{G}$ value for the ligand-COX-2complex indicated selective COX-2 inhibition for compound (E)-OXM, in contradiction of our experimentally reported results (Table 4). In the second approach, we used AM1-BCC charges from antechamber program in AMBER Tools 12 to calculate atomic charges and perform the MD simulation. In this step, we realized that the charge of an amine group in any molecule that has 
been calculated by an AM1 force field is assigned as a negatively signed number which is incorrect since amine groups, when charged, are normally cationic (Tables S6 and S7 in supplementary material). Therefore, molecules with an amine group, such as AMID and SULF, did not give logical COX-1/COX-2 binding energies and were predicted to be COX-2 selective inhibitors, contrary to experimental observations (Tables 5 and 6). Ultimately, a PM3 force field was used for the atomic-charges calculation. All compounds gave reasonable and satisfactory results in MD simulation and mm-pbsa analysis when using the PM3 force field. The detailed binding free energies for all compounds based on the PM3 charges are summarized in Tables 7 and 8.

$8 \mathbf{a}$

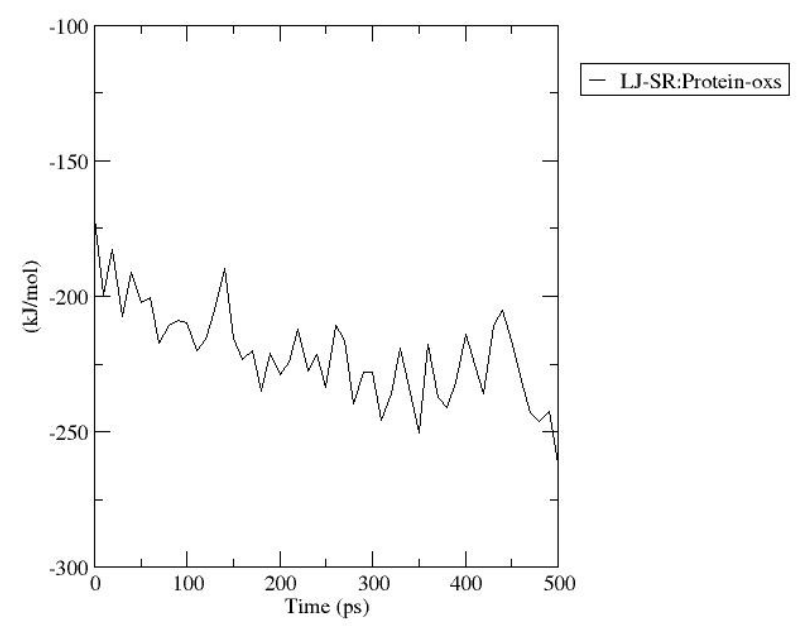

$\mathbf{8 b}$

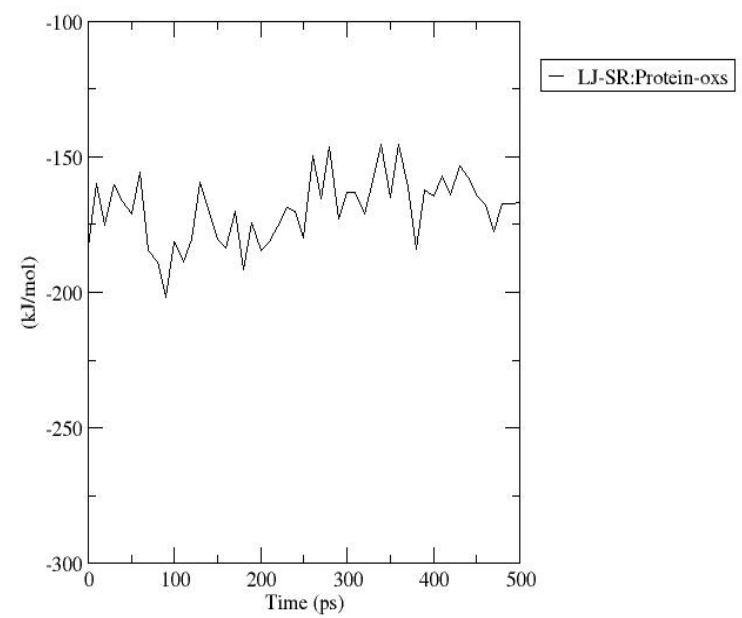

Figure 8. Short-range Lennard-Jones interaction energies for $(E)-\mathbf{O X M}$ in the active site of a) COX-1 and b) COX-2.

Plots of time-variations in Lennard-Jones and electrostatic energies and also in the number of hydrogen bonds formed in MD of $(E)-\mathbf{O X M}$ in the active site of COX-1 and COX-2 are shown in Figures 8, 9 and 10. As seen in Figure 8, Lennard-Jones interactions for $(E)-\mathbf{O X M}$ in COX-1 start at $-175 \mathrm{~kJ} / \mathrm{mol}$ and fall to $-260 \mathrm{~kJ} / \mathrm{mol}$ over $500 \mathrm{ps}$, with an average energy of $-220 \mathrm{~kJ} / \mathrm{mol}$. The Lennard-Jones interaction energies for $(E)-\mathbf{O X M}$ in COX-2 start at $-170 \mathrm{~kJ} / \mathrm{mol}$ and rise slightly over 500 ps to $-165 \mathrm{~kJ} / \mathrm{mol}$ (average energy $-170 \mathrm{~kJ} / \mathrm{mol}$ ). Clearly the Lennard-Jones energies are much lower for $(E)-\mathbf{O X M}$ in COX-1 than in COX-2 in 500 ps of MD simulation which helps to overall reduction of free energy of binding. 

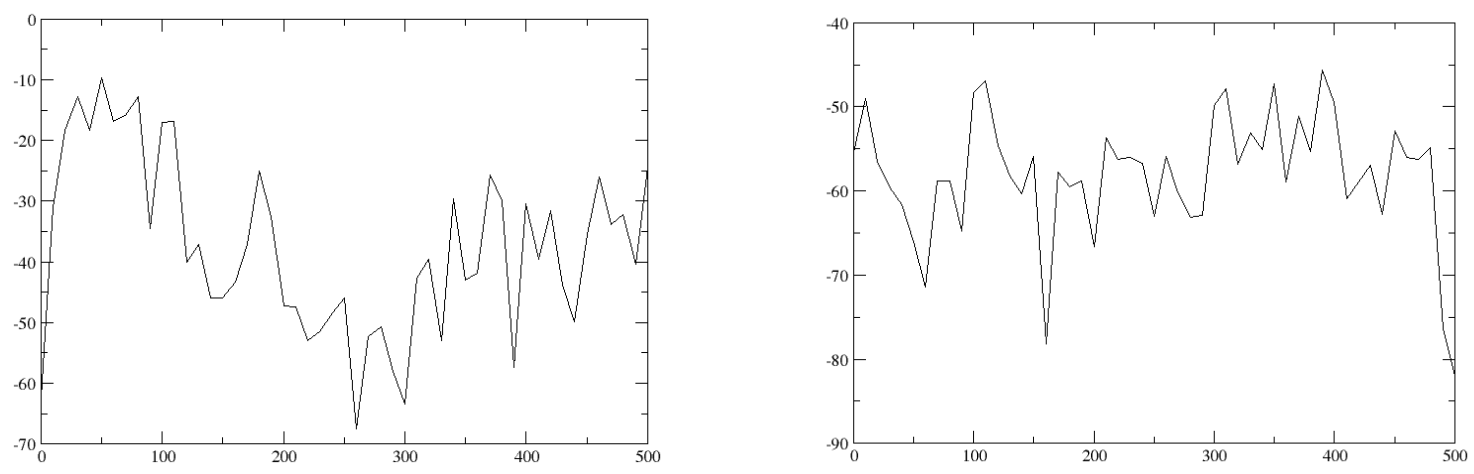

Figure 9. Electrostatic energy plot for (E)-OXM during 500 ps of simulation in the active site of a) COX1 and b) COX-2.

$10 \mathrm{a}$

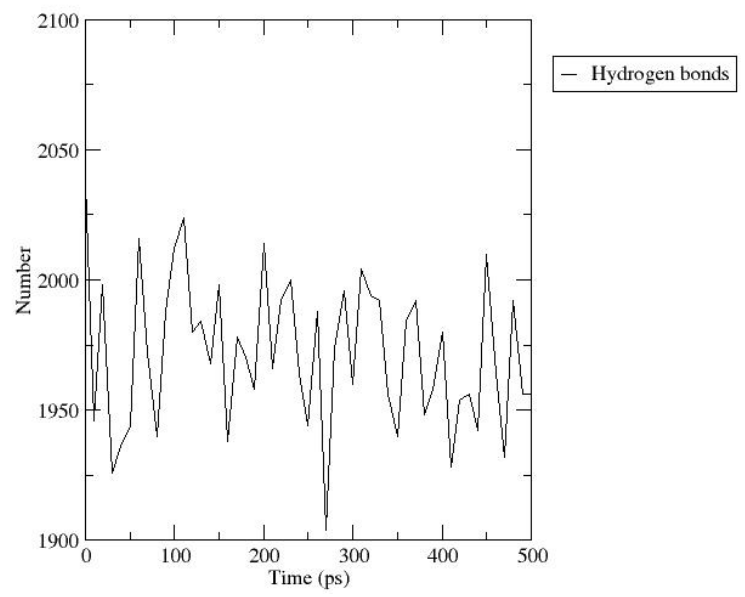

10b

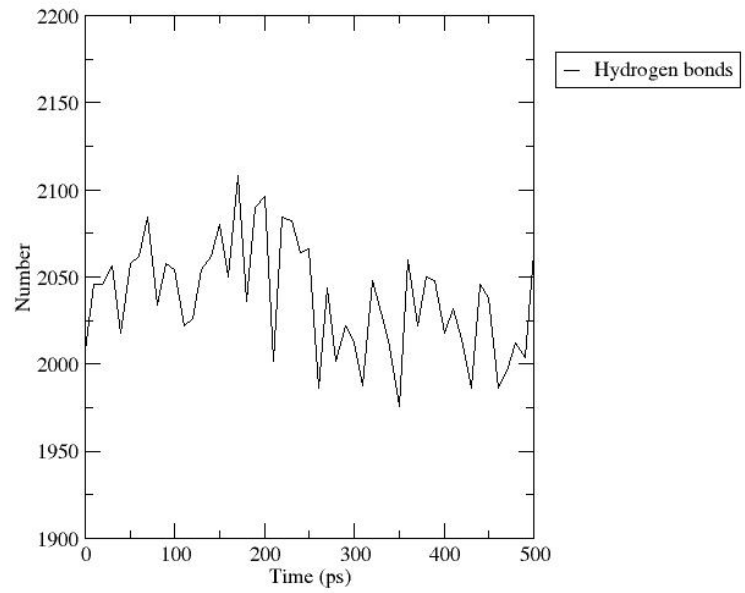

Figure 10. Number of hydrogen bonds formed during $500 \mathrm{ps}$ of simulation of compound (E)-OXM in the active site of COX-1 (left diagram) and COX-2 (right diagram).

Inversely, the electrostatic interaction energies are lower for $(E)-\mathbf{O X M}$ in COX-2 than in COX1, as is seen in Figure 9. The electrostatic energy in COX-1 starts at $-60 \mathrm{~kJ} / \mathrm{mol}$ with a rapid rise within the first $30 \mathrm{ps}$ followed by descending and ascending slopes ending at $-25 \mathrm{~kJ} / \mathrm{mol}$. During 500 ps of MD, the electrostatic energy falls below $-60 \mathrm{~kJ} / \mathrm{mol}$ in COX-1 only at two points and the average energy value is $-40 \mathrm{~kJ} / \mathrm{mol}$. The electrostatic energy is more favorable for $(E)-\mathbf{O X M}$ in COX-2, since it starts at $-55 \mathrm{~kJ} / \mathrm{mol}$ and ends at $-80 \mathrm{~kJ} / \mathrm{mol}$, with an average value of -60 $\mathrm{kJ} / \mathrm{mol}$. This shows that polar interactions are more common in COX-2 than COX-1. This fact is further confirmed by considering the number of hydrogen bonds formed between $(E)-\mathbf{O X M}$ and 
the two enzymes. As illustrated in Figure 10, the average numbers of hydrogen bonds formed at the COX-1 and COX-2 active sites are 1970 and 2020, respectively. These results are in accordance with the fact that COX-1 active site is more hydrophobic than COX-2.

$11 \mathrm{a}$

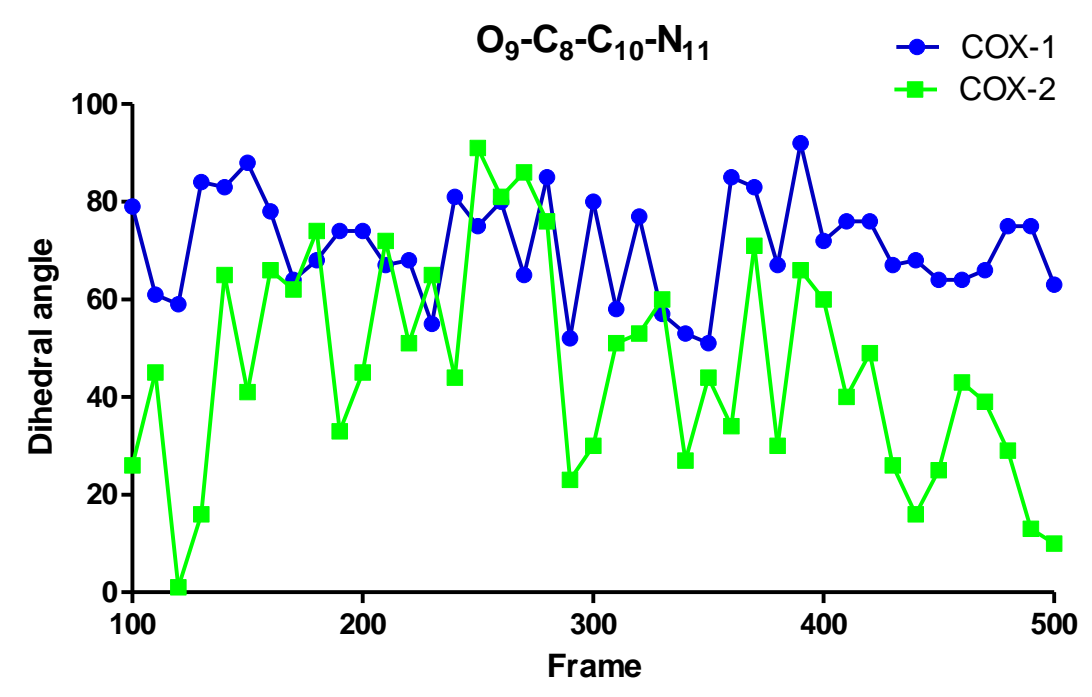

$11 b$

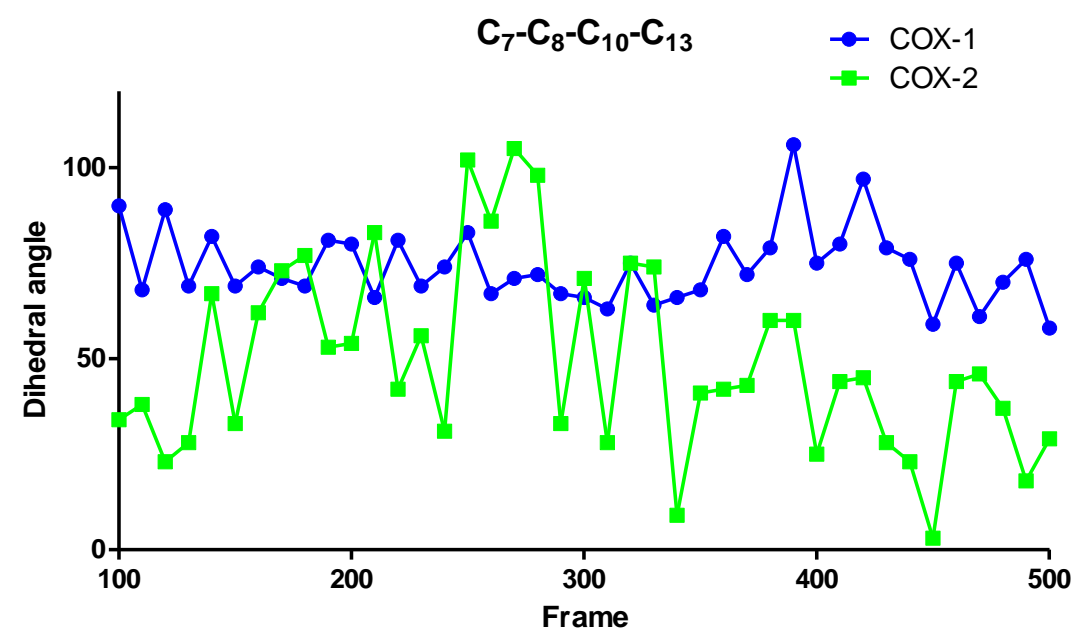

Figure 11. Variations of dihedral angles for $(E)$-OXM during the last 400 ps of MD simulation in both COX isoforms. a) Dihedral angle O9-C8-C10-N11 and b) Dihedral angle C7-C8-C10-C13

Since the COX inhibition selectivity of stilbenoids has been reported in few cases to depend on the binding conformation, we aimed to determine the variation in the two important dihedral 
angles in the 2-hydroxyiminoethanone linkage between the two aryl rings. The two key dihedral angles are $\mathrm{O} 9-\mathrm{C} 8-\mathrm{C} 10-\mathrm{N} 11$ and $\mathrm{C} 7-\mathrm{C} 8-\mathrm{C} 10-\mathrm{C} 13$ for the 2-hydroxyiminoethanone bridge between the two aryl rings. Variations in the dihedral angles O9-C8-C10-N11 and C7-C8-C10C13 during the last 400 ps are illustrated in Figure 11a and 11b, respectively. The conformations of $(E)-\mathbf{O X M}$ were extracted every 10 ps ignoring the first 100 ps. Each point in these Figures represents a specific binding conformation in COX-1 and/or COX-2 active sites. These diagrams simply show the significant difference in the $(E)-O X M$ bound conformation between COX-1 and COX-2. Both dihedral angles (O9-C8-C10-N11 and C7-C8-C10-C13) are much larger in COX-1 than in COX-2 and it means that (E)-OXM is almost in the transoid conformation when bound to the COX-1 active site and near to cisoid when bound to the COX-2 active site. This finding is in accordance with our previously reported study on how binding conformations of stilbenoids influence their COX inhibition selectivity (Irannejad, et al., 2015). This finding also led us to the conclusion that the presence of pharmacophoric groups specific for COX-2 selective inhibition, such as sulfonamide or sulfonylmethyl, in the structure of stilbenoids are not necessary but also conformational flexibility and stability is of greater importance. The results can hopefully lead us to design novel selective $\mathrm{COX}-1$ or $\mathrm{COX}-2$ stilbenoid inhibitors in the future.

\section{Conclusion}

In summary, 2-hydroxyiminoethanones are an interesting scaffold with unique geometrical properties. In this study, a recently reported COX-1 selective inhibitor with a 2hydroxyiminiethanone structure was selected for a comprehensive computational study. X-ray crystallography of this compound shows that its $(E)$-transoid conformation is stable and that there is a hydrogen bonding network between molecules in the crystal structure. Geometry optimization resulted in the most stable transoid conformation of this molecule. Results of flexible docking confirm the experimentally reported COX selectivity data. Moreover, binding free energies calculated by the MM-PBSA method are dependent on the type of force field by which atomic charges are calculated and the best results are obtained by PM3. Finally, the binding conformation of $(E)-\mathbf{O X M}$ was assessed throughout the whole molecular dynamic simulation period and its COX inhibition selectivity was shown to be conformational and not pharmacophoric dependent. 


\section{Conflict of interest}

Authors declare no conflict of interest.

\section{Acknowledgement}

This study was supported by IRAN NATIONAL SCIENCE FOUNDATION (INSF) through a grant no. 93036485. The authors thank the University of Reading for use of the Gemini diffractometer in the Chemical Analysis Facility.

\section{References}

Barbalho, P. G., Carvalho, B. D., Lopes-Cendes, I., \& Maurer-Morelli, C. V. (2016). Cyclooxygenase-1 as a Potential Therapeutic Target for Seizure Suppression: Evidences from Zebrafish Pentylenetetrazole-Seizure Model. Frontiers in Neurology, 7doi:ARTN 200

10.3389/fneur.2016.00200 Retrieved from <Go to ISI >://WOS:000387791000002

Betteridge, P. W., Carruthers, J. R., Cooper, R. I., Prout, K., \& Watkin, D. J. (2003). CRYSTALS version 12: software for guided crystal structure analysis. Journal of Applied Crystallography, 36, pp. 14871487. doi:Doi 10.1107/S0021889803021800 Retrieved from < Go to ISI>://WOS:000186682100031

Calvello, R., Lofrumento, D. D., Perrone, M. G., Cianciulli, A., Salvatore, R., Vitale, P., . . Scilimati, A. (2017). Highly Selective Cyclooxygenase-1 Inhibitors P6 and Mofezolac Counteract Inflammatory State both In Vitro and In Vivo Models of Neuroinflammation. Front Neurol, 8, p 251. doi:10.3389/fneur.2017.00251 Retrieved from https://www.ncbi.nlm.nih.gov/pubmed/28649222

Chaudhary, N., \& Aparoy, P. (2017). Deciphering the mechanism behind the varied binding activities of COXIBs through Molecular Dynamic Simulations, MM-PBSA binding energy calculations and perresidue energy decomposition studies. Journal of Biomolecular Structure \& Dynamics, 35(4), pp. 868-882. doi:10.1080/07391102.2016.1165736 Retrieved from $<$ Go to $|S|>: / /$ WOS:000399162700014

Choi, S. H., Aid, S., Caracciolo, L., Minami, S. S., Niikura, T., Matsuoka, Y., . . Bosetti, F. (2013). Cyclooxygenase- 1 inhibition reduces amyloid pathology and improves memory deficits in a mouse model of Alzheimer's disease. Journal of Neurochemistry, 124(1), pp. 59-68. doi:10.1111/jnc.12059 Retrieved from <Go to ISI>://WOS:000312132900007

Dadashpour, S., Kucukkilinc, T. T., Tan, O. U., Ozadali, K., Irannejad, H., \& Emami, S. (2015). Design, Synthesis and In Vitro Study of 5,6-Diaryl-1,2,4-triazine-3-ylthioacetate Derivatives as COX-2 and beta-Amyloid Aggregation Inhibitors. Archiv Der Pharmazie, 348(3), pp. 179-187. doi:10.1002/ardp.201400400 Retrieved from <Go to ISI >://WOS:000350502100002

Frisch, M. J., Trucks, G. W., Schlegel, H. B., Scuseria, G. E., Robb, M. A., Cheeseman, J. R., . . Fox, D. J. (2009). Gaussian 09 (Version Revision A.02). Wallingford CT: Gaussian, Inc.

Irannejad, H., Tan, O. U., Ozadali, K., Dadashpour, S., Kucukkilinc, T. T., Ahangar, N., . . Emami, S. (2015). 1,2-Diaryl-2-hydroxyiminoethanones as Dual COX-1 and beta-Amyloid Aggregation Inhibitors: Biological Evaluation and In Silico Study. Chemical Biology \& Drug Design, 85(4), pp. 494-503. doi:10.1111/cbdd.12435 Retrieved from <Go to ISI >://WOS:000351181300010 
Kakuta, H., Zheng, X. X., Oda, H., Harada, S., Sugimoto, Y., Sasaki, K., \& Tai, A. (2008). Cyclooxygenase-1selective inhibitors are attractive candidates for analgesics that do not cause gastric damage. design and in vitro/in vivo evaluation of a benzamide-type cyclooxygenase- 1 selective inhibitor. Journal of Medicinal Chemistry, 51(8), pp. 2400-2411. doi:10.1021/jm701191z Retrieved from $<$ Go to $|S|>: / / W O S: 000255105600009$

Kaur, K., Gill, J. S., Bansal, P. K., \& Deshmukh, R. (2017). Neuroinflammation - A major cause for striatal dopaminergic degeneration in Parkinson's disease. J Neurol Sci, 381, pp. 308-314. doi:10.1016/j.jns.2017.08.3251 Retrieved from https://www.ncbi.nlm.nih.gov/pubmed/28991704

Klein, C., Fischer, C., Seichter, W., Schwarzer, A., \& Weber, E. (2011). Crystal structures of benzil monoximes controlled through configurational isomerism, molecular substitution and external complexation. Crystengcomm, 13(6), pp. 1931-1938. doi:10.1039/c0ce00620c Retrieved from $<$ Go to $|S|>: / /$ WOS:000287923800034

Kumari, R., Kumar, R., Open Source Drug Discovery, C., \& Lynn, A. (2014). g_mmpbsa--a GROMACS tool for high-throughput MM-PBSA calculations. J Chem Inf Model, 54(7), pp. 1951-1962. doi:10.1021/ci500020m Retrieved from https://www.ncbi.nlm.nih.gov/pubmed/24850022

Palatinus, L., \& Chapuis, G. (2007). SUPERFLIP - a computer program for the solution of crystal structures by charge flipping in arbitrary dimensions. Journal of Applied Crystallography, 40, pp. 786-790. doi:10.1107/S0021889807029238 Retrieved from <Go to ISI >://WOS:000248077500022

Rigaku, Xcalibur/SuperNova CCD System, \& formerly Oxford Diffraction. (2012). CrysAlisPro Software System (Version Version 1.171.36.21): Rigaku Ltd, UK.

Singh, S., Awasthi, M., Pandey, V. P., \& Dwivedi, U. N. (2017). Plant derived anti-cancerous secondary metabolites as multipronged inhibitor of COX, Topo, and aromatase: molecular modeling and dynamics simulation analyses. Journal of Biomolecular Structure \& Dynamics, 35(14), pp. 30823097. doi:10.1080/07391102.2016.1241720 Retrieved from $<$ Go to $|S|>: / /$ WOS:000418477200008

Temp, F. R., Marafiga, J. R., Milanesi, L. H., Duarte, T., Rambo, L. M., Pillat, M. M., \& Mello, C. F. (2017). Cyclooxygenase- 2 inhibitors differentially attenuate pentylenetetrazolinduced seizures and increase of pro- and anti-inflammatory cytokine levels in the cerebral cortex and hippocampus of mice. European Journal of Pharmacology, 810, pp. 15-25. doi:10.1016/j.ejphar.2017.05.013 Retrieved from $<$ Go to ISI>://WOS:000407657800003

Uddin, M. J., Rao, P. N., Rahim, M. A., McDonald, R., \& Knaus, E. E. (2004). A new class of acyclic 2-alkyl1,2-diaryl (E)-olefins as selective cyclooxygenase-2 (COX-2) inhibitors. Bioorg Med Chem Lett, 14(19), pp. 4911-4914. doi:10.1016/j.bmcl.2004.07.027 Retrieved from https://www.ncbi.nlm.nih.gov/pubmed/15341950

Zheng, X. X., Oda, H., Takamatsu, K., Sugimoto, Y., Tai, A., Akaho, E., . . Sasaki, K. (2007). Analgesic agents without gastric damage: Design and synthesis of structurally simple benzenesulfonanilide-type cyclooxygenase-1-selective inhibitors. Bioorganic \& Medicinal Chemistry, 15(2), pp. 1014-1021. doi:10.1016/j.bmc.2006.10.029 Retrieved from <Go to ISI>://WOS:000243315300039 